\title{
CrystEngComm
}

Check for updates

Cite this: CrystEngComm, 2019, 21, 2105

Received 24th August 2018,

Accepted 16th October 2018

DOI: $10.1039 / \mathrm{c} 8 \mathrm{ce} 01428 \mathrm{k}$

rsc.li/crystengcomm

\section{Polymorph control in batch seeded crystallizers. A case study with paracetamol $\dagger$}

\author{
Lucrèce Nicoud, (D) Filippo Licordari and Allan S. Myerson (D)*
}

A large number of active pharmaceutical ingredients are known to crystallize in different polymorphic forms, ${ }^{1}$ i.e., in crystalline structures characterized by different packing and/ or molecular arrangement. Different polymorphs may exhibit different properties, for instance in terms of solubility, bioavailability, color, shape, hygroscopy, filterability or compactability. ${ }^{2,3}$ It is thus key to produce the target polymorph at a high purity in order to obtain the desired crystal properties and to comply with regulatory requirements. Several strategies have been investigated with a view to controlling polymorphism, including the level of supersaturation, the choice of the solvent, the use of soluble additives, seeds, and engineered surfaces. ${ }^{4-7}$ In any case, the polymorphic outcome is dictated by the relative rates of nucleation and growth of the various forms. ${ }^{6,8}$ In addition, when the solute concentration drops below the solubility of a metastable form, the polymorphic content is also affected by the kinetics of solvent-mediated transformation. The latter occurs due to the dissolution of a metastable form followed by the recrystallization of a more stable (i.e., less soluble) form as the system strives to reach equilibrium. The solventmediated transformation of a number of systems has been studied both experimentally and theoretically. ${ }^{6,9-17}$ It was found that stable forms often nucleate on crystals of a meta-

Massachusetts Institute of Technology, 77 Massachusetts Avenue, Cambridge, MA02139, USA. E-mail: myerson@mit.edu

$\dagger$ Electronic supplementary information (ESI) available: Characterization of the seeds, example of solubility measurement of form II, density measurements, calibration of the analytical tools, shape factor of form II, additional crystallization experiments, comparison of crystallization rates of form I with previous studies, impact of operating conditions on the normalized yield. See DOI: 10.1039/ c8ce01428k stable form and that the rate-limiting step (dissolution or recrystallization) depends on the system under investigation.

Seeding the crystallizer with crystals of the target polymorph is often regarded as a relatively straightforward manner to obtain the intended form. Seeding has for example been used to control the polymorphism of a steroid, ${ }^{18}$ abercarnil ${ }^{18}$ and carbamazepine. ${ }^{19}$ However, it is important to realize that seeding can help obtaining the desired polymorph only if the secondary nucleation and growth rates of the latter surpass the primary nucleation and growth rates of the unwanted polymorph(s). In addition, the polymorphic purity of the seeds plays a key role in the polymorphic outcome since small traces of an unwanted polymorph may prevent the crystallization of the desired form, as it is for example the case with ritonavir. ${ }^{20}$ Despite the large number of studies on polymorphic systems, a deep understanding of polymorph formation is still lacking and polymorph control is thus largely a matter of trial and error. Further investigations are therefore required to unravel the kinetics of polymorph formation with a view to achieving a better control of the polymorphic outcome.

In this work, we select paracetamol (acetaminophen) as a model system. In solution, paracetamol forms two polymorphs: form I, which is monoclinic and the most stable under usual temperatures, and form II, which is orthorhombic and metastable. Form II has a better compression behavior than form $\mathrm{I}^{21,22}$ but is relatively difficult to obtain and is thus not produced commercially. Three other polymorphs have been reported in the literature: form III, which was crystallized from the melt and was shown to be highly unstable, ${ }^{23}$ and forms IV and V, which were obtained at high pressure. ${ }^{24}$ Here, we target the production of form II. We start our study 
by performing batch crystallization experiments in ethanol under various concentrations, temperatures, and stirring speeds. In each case, the crystallizer is seeded with crystals of form II. We monitor the solute concentration and the polymorphic content with online infrared and Raman spectroscopy, respectively. Then, we develop a kinetic model to extract the kinetic parameters of both forms of paracetamol. Finally, we use the kinetic model to select the most suitable operating conditions to obtain form II within target specifications in terms of polymorphic purity, yield, and productivity.

\section{Materials and methods}

\subsection{Materials}

Paracetamol form I was purchased from Sigma-Aldrich (Acetaminophen BioXtra, $\geq 99 \%$ ), metacetamol from TCI America (3'-hydroxyacetanilide, $\geq 98 \%$ ) and anhydrous ethanol from Deacon Labs (Koptec 200 proof pure ethanol).

\subsection{Preparation of seeds of form II}

Crystals of paracetamol form II were prepared in the presence of metacetamol, as suggested by Agnew et $a l^{25}$ To do so, a solution containing $300 \mathrm{mg}$ paracetamol and $30 \mathrm{mg}$ metacetamol per $\mathrm{g}$ of ethanol was cooled from $50{ }^{\circ} \mathrm{C}$ to $0{ }^{\circ} \mathrm{C}$ at $75 \mathrm{rpm}$ using the set-up described in section 1.4. When the temperature reached $1^{\circ} \mathrm{C}$, the agitation was increased to $400 \mathrm{rpm}$ and $100 \mathrm{mg}$ of seeds of form II were introduced in the crystallizer. After $4 \mathrm{~h}$, the crystallization was stopped and the crystals were collected by filtration under vacuum. The crystals were dried overnight under the hood and were then sieved (Scienceware, Mini-Sieve, Micro Sieve Set, Bel-Art). Crystals comprised between the third mesh screen (number 45 according to the U.S. standard mesh scale) and the fourth mesh screen (number 60) were collected.

The aforementioned procedure requires seeding the crystallizer with pre-formed crystals of form II. The very first seeds of form II were obtained by melting commercial paracetamol on a glass slide and letting it cool down at room temperature. ${ }^{23}$ Crystallization from the melt was poorly reproducible and difficult to scale-up. In addition, the crystals of form II obtained from the melt were only stable for a few days. Therefore, solution crystallization in the presence of metacetamol was preferred for the subsequent preparations of seeds. With this procedure, no trace of paracetamol form I could be detected in the seeds by powder X-ray diffraction (PXRD), Raman spectroscopy and Fourier transform infrared (FTIR) spectroscopy, even after several months of storage. The detection limit of form I with PXRD was estimated to approximately $5 \%$. More details about the experimental characterization of the seeds are given in the ESI. +

\subsection{Solubility measurements}

1.3.1 Form I. Saturated solutions of paracetamol form I were prepared by magnetically stirring an excess of commercial paracetamol in contact with $1 \mathrm{~mL}$ ethanol. The vials were placed in a Crystal16 apparatus (Technobis Crystallization Systems) to precisely control the temperature. After $24 \mathrm{~h}$, the solution was filtered with a $0.2 \mu \mathrm{m}$ syringe filter (VWR, 13 $\mathrm{mm}$ diameter, PTFE membrane) and the filtrate was placed in tared vials. The vials were weighted immediately after introducing the filtrate and after evaporating the solvent. Ethanol was first evaporated under the hood and the last traces were removed by drying the crystals in a vacuum oven at around $60{ }^{\circ} \mathrm{C}$. Once the mass of the samples was constant over time, the solubility was computed as the mass of crystals divided by the mass of solvent that had evaporated.

1.3.2 Form II. Solubility measurements of form II were complicated by solvent-mediated transformation. To circumvent this issue, for each investigated temperature, paracetamol solutions of increasing concentrations above the solubility of form I were prepared by increments of $5 \mathrm{mg} \mathrm{g}^{-1}$. Few seeds of form II were added to these solutions and tracked by microscopy. To do so, an inverted microscope platform (Axio Observer Z.1m, Zeiss) equipped with a temperature controller was used, which allowed monitoring the behavior of several samples in parallel. For each temperature, the solubility of form II was estimated as the lowest concentration where dissolution of the seeds did not occur within the time frame of the experiment (approximately $16 \mathrm{~h}$ ). For illustrative purposes, pictures recorded during a representative experiment are shown in the ESI. $\dagger$

\subsection{Crystallization experiments}

All crystallization experiments were performed in batch mode in an EasyMax 102 Advanced Synthesis Workstation (Mettler Toledo) using a $100 \mathrm{~mL}$ two-piece glass reactor equipped with a PTFE cover and an overhead pitch-blade impeller (AlloyC22, $38 \mathrm{~mm}$ diameter, downwards). Paracetamol solutions were prepared by dissolving commercial paracetamol in ethanol at $50{ }^{\circ} \mathrm{C}$ under stirring. Then, $100 \mathrm{~mL}$ of the so-prepared solution were transfered in the EasyMax reactor. The solution was stirred at $75 \mathrm{rpm}$ and the temperature was maintained at $50{ }^{\circ} \mathrm{C}$ during $5 \mathrm{~min}$. After that, the temperature was decreased to the set temperature $\left(T=-10{ }^{\circ} \mathrm{C}, 0{ }^{\circ} \mathrm{C}\right.$ or $\left.+10{ }^{\circ} \mathrm{C}\right)$ using the steepest achievable ramp (around $4{ }^{\circ} \mathrm{C} \mathrm{min}^{-1}$ ). The solution was cooled under slow agitation (75 rpm) to avoid the formation of crystals during cooling. It was verified with online imaging that no crystals were formed during the cooling phase. Once the temperature reached $1^{\circ} \mathrm{C}$ above the set point, the agitation was increased to the set value $(N=$ 200,300 , or $400 \mathrm{rpm}$ ) and $100 \mathrm{mg}$ of seeds of form II were introduced in the crystallizer.

\subsection{Process analytics}

1.5.1 Online attenuated total reflection Fourier transform infrared spectroscopy. The concentration of paracetamol in the liquid phase was monitored by online attenuated total reflection Fourier transform infrared (ATR-FTIR) spectroscopy using a ReactIR 15 system from Mettler Toledo equipped with a $6.3 \mathrm{~mm}$ AgX DiComp immersion probe and a diamond 
as ATR crystal. At each time point, 256 spectra were recorded. Details on the calibration procedure are given in the ESI, $\dagger$ while calibration results are given in section 2.2 .

1.5.2 Online microscopy. Crystals were visualized online with high resolution microscopy using a Blaze900 probe from BlazeMetrics. The probe uses a $532 \mathrm{~nm}$ laser, has a field of view of $900 \mu \mathrm{m}$ and allows detecting crystals above approximately $2 \mu \mathrm{m}$. The image plane was set to $120 \mu \mathrm{m}$.

1.5.3 Offline Raman spectroscopy. The percentage of crystals of form II was estimated by offline Raman spectroscopy with a view to calibrating online Raman measurements. To do so, 0.5 $\mathrm{mL}$ aliquots were withdrawn from the crystallizer at different time points and dried on a filter paper. The crystals were then analyzed with a Raman WorkStation from Kaiser Optical Systems using a $20 \times$ optical lens. The exposure time was set to $1 \mathrm{~s}$ and two accumulations were collected. For each sample, measurements were taken at five different locations. The symbols and error bars in the graphs represent the average and standard deviations of those five measurements, respectively. Details on the calibration procedure are given in the ESI, $\dagger$ while calibration results are given in section 2.2.

1.5.4 Online Raman spectroscopy. Online Raman spectroscopy was used to monitor the concentration of crystals of form II as a function of time. It was implemented using a Blaze900 probe (same probe as that used for online microscopy) connected to a RamanRxn2 Hybrid Analyzer from Kaiser Optical Systems equipped with a $785 \mathrm{~nm}$ laser. The collection time was set to $45 \mathrm{~s}$ and two spectra were acquired per measurement.

The calibration was performed by regressing online Raman data of one crystallization experiment on the concentration of crystals of form II estimated independently (using ATR-FTIR to measure the total solid concentration and offline Raman to measure the fraction of form II). The regression was performed using principal component regression (PCR), which consists of applying principal component analysis (PCA) followed by multilinear regression.

Let us denote $m_{t}$ and $m_{s}$ the number of times and Raman shifts at which the Raman intensity is measured during the kinetic experiment. These data are stored in the matrix $\mathbf{M}$ (of size $m_{t} \times m_{s}$ ). Note that the number of selected Raman shifts should necessarily be smaller than the number of times at which the spectra are recorded. PCA consists in projecting the matrix $\mathbf{M}$ onto a score matrix $\mathbf{T}_{\mathbf{r}}$ of size $m_{t} \times m_{r}$ with $m_{r} \leq$ $m_{s}$. Typically, the dimension of the problem is reduced from $m_{s} \approx 10^{2}-10^{4}$ strongly correlated or even linearly dependent variables to $m_{r} \approx 2-10$ linearly independent variables, which are linear combinations of the original variables. The matrix of weights (or loadings), which regresses $\mathbf{M}$ on $\mathbf{T}_{\mathbf{r}}$ is denoted $\mathbf{W}_{\mathbf{r}}$ (of size $m_{s} \times m_{r}$ ) and is defined such that:

$$
\mathbf{T}_{\mathbf{r}}=\mathbf{M W _ { \mathbf { r } }}
$$

Note that eqn (1) can be applied to $m_{r}=m_{s}$, so that the matrices $\mathbf{T}_{\mathbf{s}}$ and $\mathbf{W}_{\mathbf{s}}$ are of size $m_{t} \times m_{s}$ and $m_{s} \times m_{s}$, respectively. The size of the problem can then be reduced to $m_{r}<$ $m_{s}$ simply by defining $\mathbf{T}_{\mathbf{r}}$ and $\mathbf{W}_{\mathbf{r}}$ as the first $m_{r}$ columns of $\mathbf{T}_{\mathbf{S}}$ and $\mathbf{W}_{\mathbf{s}}$, respectively. Once the matrices $\mathbf{W}_{\mathbf{r}}$ and $\mathbf{T}_{\mathbf{r}}$ have been determined, the matrix $\mathbf{M}$ is regressed on the vector $\mathbf{c}^{\mathrm{II}}$ (of size $m_{t} \times 1$ ) containing the known concentrations of crystals of form II. This can be mathematically written as:

$$
\mathbf{c}^{\mathrm{II}}=\mathbf{M p}
$$

where $\mathbf{p}$ is the regression vector (of size $m_{s} \times 1$ ) and can be estimated as:

$$
\mathbf{p}=\mathbf{W}_{\mathbf{r}}\left(\mathbf{T}_{\mathbf{r}}^{\prime} \mathbf{T}_{\mathbf{r}}\right)^{-1} \mathbf{T}_{\mathbf{r}}^{\prime} \mathbf{c}^{\mathrm{II}}
$$

In the case where the data are not passing through the origin, a constant coefficient must be added to eqn (2). This can be implemented by rewriting the equation as follows:

$$
\mathbf{c}^{\mathrm{II}}=\mathbf{N q}
$$

where $\mathbf{N}$ is obtained by adding a column of ones to the left of the matrix $\mathbf{M}$, so that $\mathbf{N}$ is of size $m_{t} \times\left(m_{s}+1\right)$. The vector $\mathbf{q}$ of size $\left(m_{s}+1\right) \times 1$ is instead obtained by adding the scalar term mean $\left(\mathbf{c}_{\mathbf{I I}}\right)-\mathbf{M p}$ on top of the vector $\mathbf{p}$. Once the vector $\mathbf{q}$ has been determined from the regression experiment, the concentration of form II can be computed for any ensemble of Raman spectra stored in a new matrix $\mathbf{M}$ by using eqn (4).

\subsection{Kinetic model}

The kinetic model used in this paper accounts for the nucleation and growth of form I as well as for the nucleation, growth and dissolution of form II. The selected expressions of the nucleation $(B)$, growth $(G)$ and dissolution $(D)$ rates are given in Table 1 . The supersaturation derives from a difference in chemical potential and is thus best described as the logarithm of the ratio between the actual concentration in the liquid phase (denoted $c^{\text {liq }}$ ) and the concentration at saturation, i.e., the solubility (denoted $c_{\mathrm{I}}^{*}$ and $c_{\mathrm{II}}^{*}$ for forms I and II, respectively). A linearization of the dissolution rate was employed because the solute concentration is close to the solubility of form II during the dissolution process.

The nucleation rates are assumed to be proportional to the second order moment (denoted $\mu_{2}$ ) of form I or form II depending on the type of nucleation. The second order moment is proportional to the total crystal surface and is defined rigorously later on (see eqn (7) and (8)), while some additional comments on the expression of the nucleation rates are given here. Considering form II first, secondary nucleation is expected to be dominant with respect to primary nucleation since the crystallizer is seeded with crystals of form II. Accordingly, the nucleation rate of form II is considered to be proportional to $\mu_{2}^{\mathrm{II}}$ (see $B_{\mathrm{s}}^{\mathrm{II}}$ in Table 1). Note that such lumped expression is sometimes assumed to average contributions from both primary and secondary nucleation in a coarse-grained manner. On the other hand, it is necessary to explicitly account for the primary nucleation of form I in order to explain its appearance in the crystallizer (setting $\mu_{2}^{\mathrm{I}}=0$ at time 0 would indeed 
Table 1 Expressions of the nucleation, growth and dissolution rates

\begin{tabular}{|c|c|}
\hline \multicolumn{2}{|l|}{ Form I } \\
\hline Cross-nucleation & $B_{\mathrm{c}}^{\mathrm{I}}=k_{\mathrm{c}}^{\mathrm{I}}\left[\ln \left(\frac{c^{\mathrm{liq}}}{c_{1}^{*}}\right)\right]^{n_{\mathrm{c}}} \mu_{2}^{\mathrm{II}}$ \\
\hline Secondary nucleation & $B_{\mathrm{s}}^{\mathrm{I}}=k_{\mathrm{s}}^{\mathrm{I}}$ \\
\hline Growth & $G^{\mathrm{I}}=k_{\mathrm{g}}^{\mathrm{I}}\left[\ln \left(\frac{c^{\mathrm{liq}}}{c_{\mathrm{I}}^{*}}\right)\right]^{n_{\mathrm{g}}}$ \\
\hline \multicolumn{2}{|l|}{ Form II } \\
\hline Secondary nucleation $\left(c^{\text {liq }} \geq c_{\mathrm{II}}^{*}\right)$ & $B_{\mathrm{s}}^{\mathrm{II}}=k_{\mathrm{s}}^{\mathrm{II}}\left[\ln \left(\frac{c^{\mathrm{liq}}}{c_{\mathrm{II}}^{*}}\right)\right]^{n_{\mathrm{s}}} \mu_{2}^{\mathrm{II}}$ \\
\hline $\operatorname{Growth}\left(c^{\text {liq }} \geq c_{\mathrm{II}}^{*}\right)$ & $G^{\mathrm{II}}=k_{\mathrm{g}}^{\mathrm{II}}\left[\ln \left(\frac{c^{\mathrm{liq}}}{c_{\mathrm{H}}^{*}}\right)\right]^{n_{\mathrm{g}}}$ \\
\hline Dissolution $\left(c^{\text {liq }}<c_{\mathrm{II}}^{*}\right)$ & $D^{\mathrm{II}}=k_{\mathrm{d}}^{\mathrm{II}} \frac{c^{\mathrm{liq}}-c_{\|}^{*}}{c_{\|}^{*}}$ \\
\hline
\end{tabular}

otherwise result in the absence of form I all along the crystallization process). True homogeneous primary nucleation is often considered unrealistic in finite size vessels, so that heterogeneous nucleation on surfaces is regarded as the predominant mechanism of primary nucleation. A surface that is highly likely to promote the nucleation of form I is the surface exposed by crystals of form II. Therefore, the primary nucleation of form I is considered here to be proportional to the surface of crystals of form II (see $B_{\mathrm{c}}^{\mathrm{I}}$ in Table 1). This phenomenon is referred to as cross-nucleation and has been reported for several systems in the literature, including ROY, ${ }^{26}$ L-glutamic acid, ${ }^{11,14,27}$ D-mannitol, ${ }^{28,29}$ and polypivalolactone. ${ }^{30}$ Besides cross-nucleation, form I also appears by secondary nucleation, with an expression similar to that employed for form II (see $B_{\mathrm{s}}^{\mathrm{I}}$ in Table 1).

In Table 1 , the parameters $k$ are temperature dependent rate constants, while the exponents $n$ are assumed to be temperature independent. The indexes $\mathrm{c}, \mathrm{s}, \mathrm{g}$ and $\mathrm{d}$ refer to cross-nucleation, secondary nucleation, growth and dissolution, respectively. For the sake of simplicity, it is assumed that $n_{\mathrm{s}}$ and $n_{\mathrm{g}}$ are identical for the two forms.

In a batch crystallizer, the population balance equations (PBE) describing the time evolution of the concentration of crystals of form I and II are written as follows:

$$
\begin{gathered}
\frac{\partial n^{\mathrm{I}}}{\partial t}+\frac{\partial\left(G^{\mathrm{I}} n^{\mathrm{I}}\right)}{\partial L}=\left(B_{\mathrm{c}}^{\mathrm{I}}+B_{\mathrm{s}}^{\mathrm{I}}\right) \times \delta\left(L-L_{0}\right) \\
\frac{\partial n^{\mathrm{II}}}{\partial t}+\frac{\partial\left(G^{\mathrm{II}} n^{\mathrm{II}}\right)}{\partial L}+\frac{\partial\left(D^{\mathrm{II}} n^{\mathrm{II}}\right)}{\partial L}=\left(B_{\mathrm{s}}^{\mathrm{II}}+D^{\mathrm{II}} n_{\bullet}^{\mathrm{II}}\right) \times \delta\left(L-L_{0}\right)
\end{gathered}
$$

where $t$ denotes the time and $L$ the characteristic crystal size. The concentration of crystals comprised between $L$ and $L+$ $\mathrm{d} L$, namely $n=n(t, L)$, is expressed in $\# \mathrm{~m}^{-4}$. The growth and dissolution rates are assumed to be size independent. The symbol $\delta$ represents the Dirac delta function and indicates that nucleation produces crystals of size $L_{0}$ only. On the other hand, dissolution reduces the size of crystals of form II. When a crystal of form II reaches $L_{0}$, it disappears and goes back to the liquid phase. The concentration of crystals of form II of size $L_{0}$, i.e., $n^{\mathrm{II}}\left(t, L=L_{0}\right)$, is denoted $n_{\bullet}^{\mathrm{II}}$.

In this work, the PBE were solved by using the methods of moments. The moments of order $j$ of the crystal size distributions of both forms are defined as follows:

$$
\begin{aligned}
\mu_{j}^{\mathrm{I}} & =\int_{0}^{\infty} L^{j} n^{\mathrm{I}}(t, L) \mathrm{d} L \\
\mu_{j}^{\mathrm{II}} & =\int_{0}^{\infty} L^{j} n^{\mathrm{II}}(t, L) \mathrm{d} L
\end{aligned}
$$

By applying the above definitions, the PBE given in eqn (5) and (6) are written as:

$$
\begin{gathered}
\frac{\mathrm{d} \mu_{j}^{\mathrm{I}}}{\mathrm{d} t}=j G^{\mathrm{I}} \mu_{j-1}^{\mathrm{I}}+\left(B_{\mathrm{c}}^{\mathrm{I}}+B_{\mathrm{s}}^{\mathrm{I}}\right) L_{0}^{j} \\
\frac{\mathrm{d} \mu_{j}^{\mathrm{II}}}{\mathrm{d} t}=j G^{\mathrm{II}} \mu_{j-1}^{\mathrm{II}}+\left(B_{s}^{\mathrm{II}}+D^{\mathrm{II}} n_{\cdot}^{\mathrm{II}}\right) L_{0}^{j}
\end{gathered}
$$

These equations are coupled to a mass balance on the solute:

$$
\frac{\mathrm{dc}}{\mathrm{dt}}=-3 k_{\mathrm{v}}^{\mathrm{I}} \rho^{\mathrm{I}} G^{\mathrm{I}} \mu_{2}^{\mathrm{I}}-3 k_{\mathrm{v}}^{\mathrm{II}} \rho^{\mathrm{II}} G^{\mathrm{II}} \mu_{2}^{\mathrm{II}}
$$

where $k_{v}^{i}$ and $\rho^{i}$ denote the shape factor and crystal density of form $i$, respectively. The initial conditions are given by $c^{\mathrm{liq}}(t=0)$ $=c_{0}^{\text {liq }}, n^{\mathrm{I}}(t=0, L)=0$, and $n^{\mathrm{II}}(t=0, L)=n_{\text {seeds }}^{\mathrm{II}}(L)$. The concentrations of crystals of form I and II are computed from:

$$
\begin{aligned}
c^{\mathrm{I}} & =k_{\mathrm{v}}^{\mathrm{I}} \rho^{\mathrm{I}} \mu_{3}^{\mathrm{I}} \\
c^{\mathrm{II}} & =k_{\mathrm{v}}^{\mathrm{II}} \rho^{\mathrm{II}} \mu_{3}^{\mathrm{II}}
\end{aligned}
$$

Eqn (9)-(11) are straightforward to solve in the absence of dissolution. However, when solvent-mediated transformation occurs, it is necessary to estimate $n_{\bullet}^{\mathrm{II}}$, which is not directly obtained from the moment equations. Here, we estimate $n_{\bullet}^{\text {II }}$ according to the method employed by Cornel et al. ${ }^{9}$ This method relies on the reconstruction of the crystal size distribution at the time where dissolution starts by using a Laplace based technique previously derived by Qamar et al. ${ }^{31}$ Briefly, the idea is to first solve the moment equations until $t_{\mathrm{d}}$, the time when dissolution starts, so as to obtain the time dependence of the nucleation and growth rates. Then, the size distribution of crystals of form II at time $t_{\mathrm{d}}$ can be computed as:

$$
n^{\mathrm{II}}\left(t_{\mathrm{d}}, L\right)=n_{0}^{\mathrm{II}}\left(L-u^{\mathrm{II}}\left(t_{\mathrm{d}}\right)\right)+r^{\mathrm{II}}(L)
$$

with:

$$
u^{\mathrm{II}}\left(t_{\mathrm{d}}\right)=\int_{0}^{t_{\mathrm{d}}} G^{\mathrm{II}}(\tau) \mathrm{d} \tau
$$




$$
r^{\mathrm{II}}(L)= \begin{cases}\frac{B_{\mathrm{s}}^{\mathrm{II}}(\xi)}{G^{\mathrm{II}}(\xi)} & \text { if }\left(L-L_{0}\right) \in\left[0, u^{\mathrm{II}}\left(t_{\mathrm{d}}\right)\right] \\ 0 & \text { otherwise }\end{cases}
$$

where $\xi$ is the root of the following equation:

$$
\int_{\xi}^{t_{\mathrm{d}}} G^{\mathrm{II}}(\tau) \mathrm{d} \tau-\left(L-L_{0}\right)=0
$$

which can, for instance, be solved by using Newton's formula.

The first term in eqn (14) describes the growth of seeds, while the second one accounts for nucleation and growth of newly formed crystals. Once the size distribution of crystals of form II is computed at the moment where dissolution starts, the concentration of crystals of form II of size $L_{0}$ at any time $t \geq t_{\mathrm{d}}$ can be computed as $n_{\bullet}^{\mathrm{II}}=n^{\mathrm{II}}\left(t, L=L_{0}\right)=n^{\mathrm{II}}\left(t_{\mathrm{d}}\right.$, $\left.L_{\bullet}\right)$, where the length $L_{\bullet}(t)$ is computed by integrating $\mathrm{d} L_{0} / \mathrm{d} t$ $=\left|D^{\mathrm{II}}(t)\right|$ with the initial condition $L_{\bullet}(t=0)=L_{0}$. Indeed, since dissolution simply shifts the crystal size distribution to lower sizes, it is equivalent to keep $L=L_{0}$ and increase the time $t$, or keep $t=t_{\mathrm{d}}$ and increase the length $L_{\bullet}$ at a rate equal to the dissolution rate.

The moment equations were solved using concentrations in $\mathrm{kg}$ of paracetamol per cubic meter of suspension. Density measurements were performed at various concentrations and temperatures to allow the conversion to $\mathrm{kg}$ of paracetamol per $\mathrm{kg}$ of ethanol. The results of density measurements are presented in the ESI.†

\section{Results and discussion}

\subsection{Solubility}

Since solubility dictates the thermodynamics of crystallization, we started our study by estimating the solubility of both forms of paracetamol. The measured solubility values of form I and form II in ethanol are shown as a function of temperature with blue and red circles, respectively, in Fig. 1. These data were described by a thermodynamic model in our previous article. ${ }^{32}$ Here, we simply fit the experimental data with the following exponential expressions:

$$
\left\{\begin{array}{l}
c_{1}^{*}=0.702 \exp (0.019 T) \\
c_{\mathrm{II}}^{*}=0.593 \exp (0.020 T)
\end{array}\right.
$$

where $c_{\mathrm{I}}^{*}$ and $c_{\mathrm{II}}^{*}$ are the solubilities of form I and II, respectively, expressed in $\mathrm{mg} \mathrm{g}_{\mathrm{EtOH}}{ }^{-1}$, while $T$ is the temperature in Kelvin. The results of the fitting are represented by solid lines in Fig. 1.

It is seen in Fig. 1 that form I is the most stable over the entire range of temperatures investigated. In addition, the difference in solubility between the two forms decreases at lower temperatures. This suggests that the crystallization of form II is thermodynamically more favored at low temperatures than at high temperatures.

\subsection{Calibration of process analytical tools}

Process analytical technologies (PAT) are increasingly used for process monitoring and control, both at the laboratory and industrial scales. ${ }^{33}$ In this work, ATR-FTIR and Raman spectroscopy were used to quantify the solute concentration and the concentration of form II, respectively, as a function of time. The use of such analytical tools requires first proper calibration, whose results are reported in this section.

2.2.1 Online ATR-FTIR. ATR-FTIR probes allow monitoring solute concentrations in the liquid phase independently of the presence of solids due to the low penetration depth of the IR beam (in the order of few microns). Therefore, in the case of binary systems, i.e., a single solute dissolved in a single solvent, calibration procedures of ATR-FTIR spectroscopy are rather straightforward. ${ }^{11,34,35}$ Here, the concentration of dissolved paracetamol was correlated to the height of the peak at $1514 \mathrm{~cm}^{-1}$ after subtracting the intensity at 1801 $\mathrm{cm}^{-1}$, which corresponds to the intensity of the baseline. More details about the calibration procedure are given in the ESI, $\dagger$ while the results of the linear regressions at the three considered temperatures are given below:

$$
\begin{cases}T=-10^{\circ} \mathrm{C} & \mathscr{A}_{1514}=2.00 \times 10^{-3} c^{\text {liq }}+0.205 \\ T=0{ }^{\circ} \mathrm{C} & \mathscr{A}_{1514}=1.98 \times 10^{-3} c^{\text {liq }}+0.197 \\ T=+10^{\circ} \mathrm{C} & \mathscr{A}_{1514}=1.96 \times 10^{-3} c^{\text {liq }}+0.190\end{cases}
$$

where $\mathscr{A}_{1514}$ denotes the absorbance at $1514 \mathrm{~cm}^{-1}$ and $c^{\text {liq }}$ is the solute concentration in $\mathrm{mg} \mathrm{g}_{\mathrm{EtOH}}{ }^{-1}$.

2.2.2 Offline Raman. Offline Raman analysis on dried solid samples was performed with a view to calibrating the online Raman probe. This in turn required prior calibration of the offline Raman instrument. Details about the calibration procedure are given in the ESI, $\uparrow$ while the calibration result is given here:

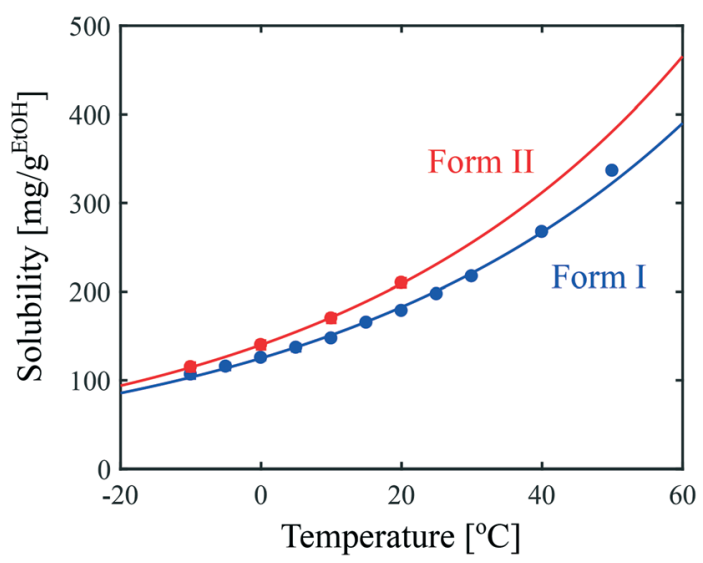

Fig. 1 Solubility of paracetamol form I and form II in ethanol as a function of temperature. Symbols represent experimental data and lines correspond to the fits given in eqn (18). 


$$
\frac{A_{454}}{A_{454}+A_{465}}=8.0 \times 10^{-3} f^{\mathrm{II}}+0.12
$$

where $A_{454}$ and $A_{465}$ denote the areas under the peaks at 454 $\mathrm{cm}^{-1}$ and $465 \mathrm{~cm}^{-1}$, respectively, obtained after baseline subtraction, while $f^{\mathrm{II}}$ denotes the mass percentage of form II.

As a comparison, the following calibration has been reported previously in the literature: $A_{454} / A_{465}=482 /\left(100-f_{\text {II }}\right)$ - 0.324. ${ }^{36}$ This expression has the disadvantage to diverge when the percentage of form II tends towards 100\%, and eqn (20) was thus preferred.

2.2.3 Online Raman. The analysis of online Raman data is often substantially more complex than that of online IR data because the Raman signal depends on many factors including the composition of the solid and liquid phases as well as the size and shape of the suspended crystals. Although in some simple cases a monovariate analysis was shown sufficient to correlate Raman spectra to the polymorphic composition, multivariate analysis is quite often required. ${ }^{10,37-39}$ Unraveling the impact of each factor (e.g., solid composition, liquid composition, crystal size, crystal shape, temperature) and their combination on Raman spectra is extremely tedious. In this work, we investigate the possibility to regress calibration parameters on one single kinetic experiment. This experiment has to be selected so as to allow the observation of a change in polymorphic composition over time. Here, the following operating parameters were chosen: $T=0{ }^{\circ} \mathrm{C}, c_{0}^{\text {liq }}=$ $300 \mathrm{mg} \mathrm{g}_{\mathrm{EtOH}}{ }^{-1}, N=400 \mathrm{rpm}$. Under these conditions, a sufficient amount of form II could be obtained by seeding the crystallizer with crystals of form II. Then, a change in polymorphism occurred due to solvent-mediated transformation. This is illustrated in Fig. 2, where it is seen that plate-like crystals are formed at the beginning of the experiment, which are characteristic of form II. On the other hand, after $6 \mathrm{~h}$, more rounded crystals are observed, characteristic of form I.

The Raman spectra at the beginning and at the end of the experiment are shown in the ESI. $\dagger$ Several peaks and peak ratios were tracked as a function of time in an attempt to rationalize the data, i.e., identify some features representing the increase in solid content and change in solid composition. However, such simple analysis was largely unsuccessful.

Exploratory data analysis can provide useful qualitative information on the main characteristics of online measurements. Here, principal component analysis (PCA) was performed on the online Raman data and the results are shown in Fig. 3. It is usually recommended to pre-process the spectra before applying statistical analysis in order to reduce the effects unrelated to the property of interest (e.g., slight fluorescence and laser fluctuations), so that pertinent changes can be better observed. Various pre-processing approaches have been used in the literature, such as the normalization, smoothing or computation of derivatives of experimental spectra. ${ }^{40,41}$ The results of Fig. 3 were obtained considering first derivatives (D1) followed by standard nor- mal variate (SNV) treatment. The latter correction consists in subtracting the mean of the spectrum and subsequently dividing it by the standard deviation. Fig. 3(a) shows the scores of the first four principal components as a function of time together with the explained percentage of variance. It is generally difficult to relate principal components to physical quantities. Nevertheless, it is seen that the trend of the first principal component $\left(\mathrm{PC}_{1}\right)$ somehow resembles the expected trend of the concentration of crystals of form II: it increases due to crystallization, reaches a maximum, and then decreases due to transformation into form $\mathrm{I}$. On the other hand, the trend of the second principal component $\left(\mathrm{PC}_{2}\right)$ is not too far from the expected trend of the concentration in the liquid phase, with a sharp decrease at the beginning followed by a quite long plateau due to the transformation. In addition, it is seen that the first three principal components describe most of the variance $(94.04 \%)$, so that PCA allows reducing the size of the system from 1800 recorded wavelengths to 3 principal components. The scores are plotted as a function of the three first principal components in Fig. 3(b). In this graph, each symbol represents a different time point and the arrows indicate the time direction. This representation allows the identification of three phases, denoted (1), (2), and (3), further discussed later on.

After principal component analysis, the online Raman data were regressed with a multilinear model on the concentration of form II determined independently from online ATR-FTIR and offline Raman data. While online ATR-FTIR provides an estimate of the solute concentration (and thus of the total solid concentration by difference), offline Raman measurements provide an estimate of the fraction of form II in dried solid samples. The combination of the two techniques therefore allows assessing the absolute concentration of crystals of form II. The regression vector was determined by PCR, as described in section 1.5.4. The quality of the regression can be appreciated in Fig. 4(a), where the online data are represented by dots and offline data by circles. Two pre-processing methods have been employed, namely SNV (purple dots) and first derivatives
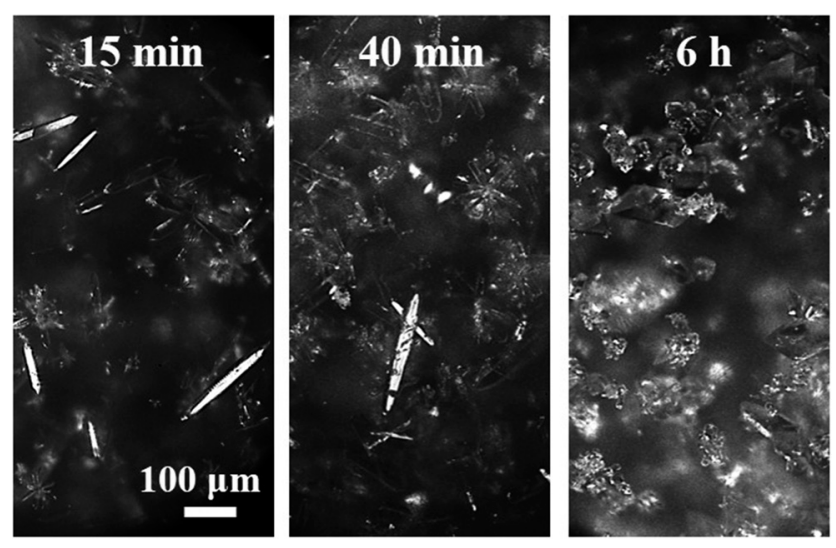

Fig. 2 Online images recorded during the seeded crystallization of form II with the following operating conditions: $T=0^{\circ} \mathrm{C}, c_{0}^{\text {liq }}=300$ $\mathrm{mg} \mathrm{g}_{\mathrm{EtOH}}{ }^{-1}, N=400 \mathrm{rpm}$. 
(a)
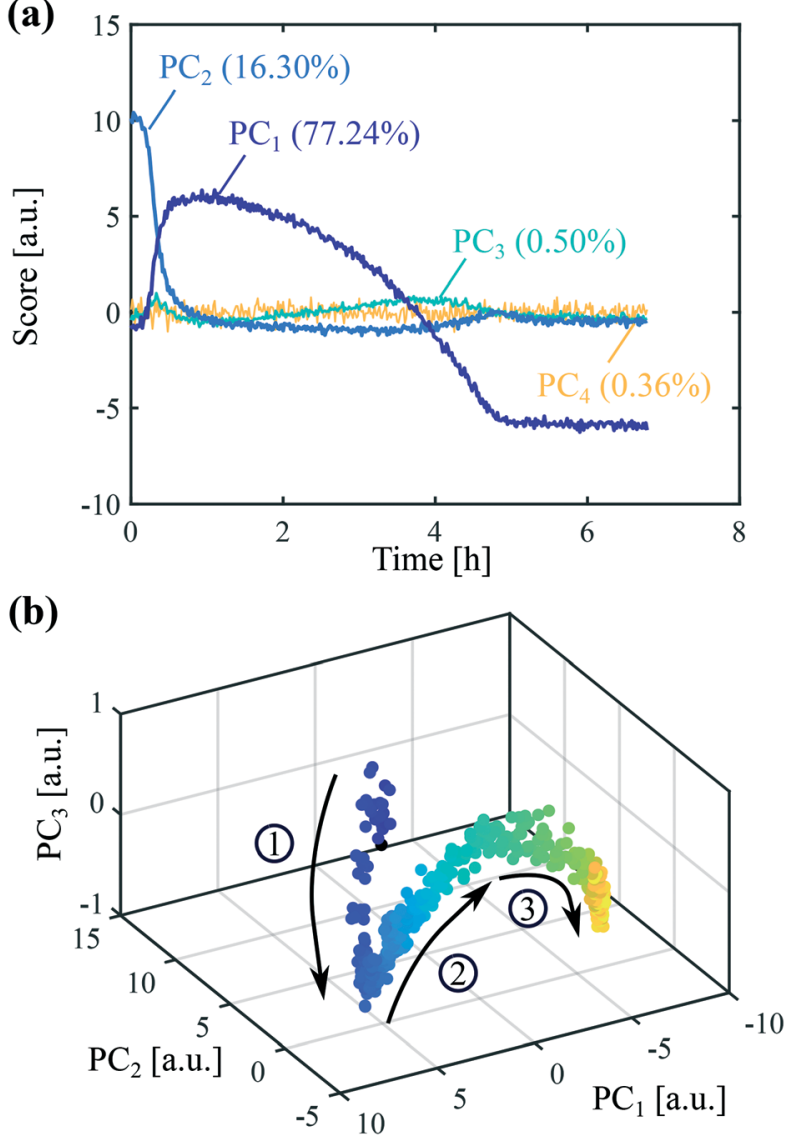

Fig. 3 Principal component analysis on a seeded crystallization experiment. The following operating conditions were employed: $T=$ $0{ }^{\circ} \mathrm{C}, \mathrm{C}_{0}^{\text {liq }}=300 \mathrm{mg} \mathrm{g}_{\mathrm{EtOH}}{ }^{-1}, N=400 \mathrm{rpm}$. (a) Scores of the first four principal components as a function of time together with their percentage of explained variance. (b) Scores over time in a three dimensional representation. Arrows indicate the time direction.

followed by SNV (red dots). It can be seen that a good regression can be obtained in both cases.

Then, the validity of the calibration was assessed by its ability to determine the concentration of form II in other conditions. To do so, three validation experiments were performed, where in each of them one operating parameter was varied as compared to the regression experiment. The results are presented in Fig. 4(b-d). In panel (b), the concentration was decreased from $300 \mathrm{mg} \mathrm{g}_{\mathrm{EtOH}}{ }^{-1}$ to $250 \mathrm{mg}$ $\mathrm{g}_{\mathrm{EtOH}}{ }^{-1}$. In panel (c), the temperature was decreased from $0{ }^{\circ} \mathrm{C}$ to $-10{ }^{\circ} \mathrm{C}$. In panel (d), the stirring speed was decreased from $400 \mathrm{rpm}$ to $300 \mathrm{rpm}$. In each case, the same two pre-processing methods as those used for the regression, namely SNV and first derivative followed by SNV, were tested. It is seen that the calculations of the online concentration of form II are in very good agreement with the offline measurements in the case where the signals were pre-processed using the first derivatives followed by SNV. As a comparison, the calculations are less accurate when using SNV alone. Therefore, the method of first derivatives combined with SNV was selected to analyze subsequent data.
The results of Fig. 4 validate the statistical model used to process online Raman data.

\subsection{Crystallization kinetics}

Batch crystallization experiments were performed in ethanol at various concentrations (from $250 \mathrm{mg} \mathrm{g}_{\mathrm{EtOH}}{ }^{-1}$ to $300 \mathrm{mg}$ $\mathrm{g}_{\mathrm{EtOH}}{ }^{-1}$ ), various temperatures (from $-10{ }^{\circ} \mathrm{C}$ to $+10{ }^{\circ} \mathrm{C}$ ), and various stirring speeds (from $200 \mathrm{rpm}$ to $400 \mathrm{rpm}$ ). In each case, the crystallizer was seeded with crystals of form II. Experimental results are presented with dots in Fig. 5. The concentration of paracetamol in the liquid phase was determined by online ATR-FTIR and is shown in black. The concentration of crystals of form II was determined by online Raman spectroscopy using the multilinear model described
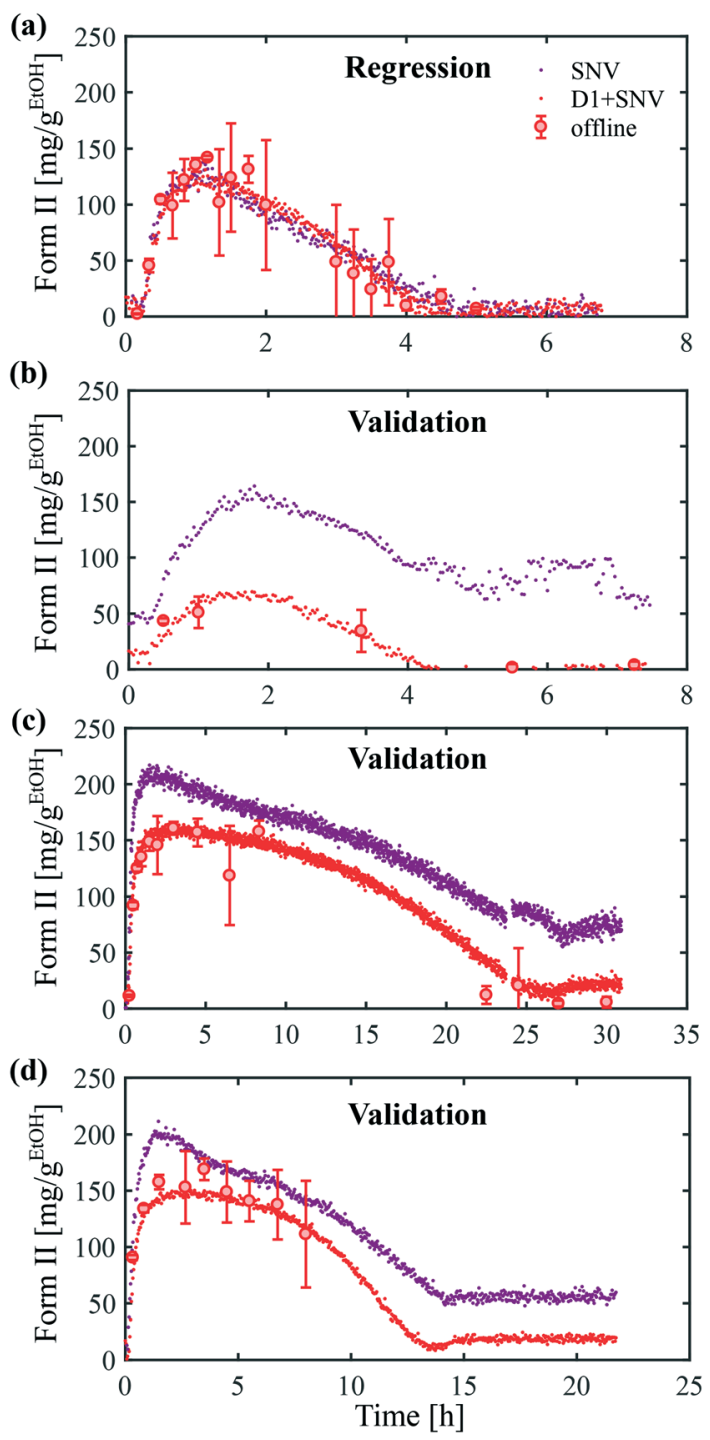

Fig. 4 Online Raman calibration. (a) Regression experiment performed with the following operating parameters: $c_{0}^{\text {liq }}=300 \mathrm{mg} \mathrm{g}_{\mathrm{EtOH}}{ }^{-1}, T=$ $0{ }^{\circ} \mathrm{C}, N=400 \mathrm{rpm}(\mathrm{b}-\mathrm{d})$ validation experiments. In each case, only one parameter was varied, namely: (b) $C_{0}^{\text {liq }}=250 \mathrm{mg} \mathrm{g}_{\mathrm{EtOH}}{ }^{-1}$, (c) $T=-10{ }^{\circ} \mathrm{C}$, (d) $N=300 \mathrm{rpm}$. 
in the previous section and is shown in red. Finally, the concentration of crystals of form I was determined by a mass balance knowing the initial concentration, the solute concentration and the concentration of crystals of form II, and is shown in blue.

Although the time frame of crystallization varies greatly between the different conditions, all the experiments share common features, with three distinct phases. During the first phase, the concentration in the liquid phase drops due to the crystallization of form II (in parallel to the crystallization of form I in some cases). Then, the crystals of form II dissolve and recrystallize as form I. During this transformation, the concentration in the liquid phase stays constant at the solubility of form II. Finally, the concentration in the liquid phase drops toward the solubility of form I due to the further crystallization of form I. These three phases correspond to the phases (1), (2), and (3) identified in Fig. 3(b).

The data presented in Fig. 5(a) correspond to a repetition of the experiment presented in Fig. 4, and thus show the good reproducibility of the experiments. It is seen in Fig. 5(ac) that a decrease in the initial solute concentration from 300 $\mathrm{mg} \mathrm{g}_{\mathrm{EtOH}}{ }^{-1}$ to $250 \mathrm{mg} \mathrm{g}_{\mathrm{EtOH}}{ }^{-1}$ slows down phase (1), i.e., it takes more time to reach the solubility of form II. This is due to a decrease in the supersaturation. In addition, it is observed that for each concentration, after $1 \mathrm{~h}$ of cooling (i.e., approximately when $c^{\text {liq }}$ approaches $c_{\mathrm{II}}^{*}$ ), the fraction of form II exceeds $60 \%$. This suggests that the initial concentration has only a weak impact on the polymorphic content.

Considering now the data shown in Fig. 5(d-f), it is seen that temperature has a very strong impact, not only on the time scale of the experiment (from $1.5 \mathrm{~h}$ at $+10{ }^{\circ} \mathrm{C}$ to $30 \mathrm{~h}$ at $-10{ }^{\circ} \mathrm{C}$ ), but also on the polymorphic content. In particular, form II can be obtained at a decent concentration only at $-10{ }^{\circ} \mathrm{C}$ and $0{ }^{\circ} \mathrm{C}$. At $+10{ }^{\circ} \mathrm{C}$, the crystallization of form I is so fast that seeding is not sufficient to induce the crystallization of form II. It is already anticipated from these data that the differences in solubility reported in Fig. 1 cannot explain such drastic changes in the polymorphic outcome. A more detailed kinetic analysis is performed in the next section.

Finally, the results shown in Fig. $5(\mathrm{~g}-\mathrm{i})$ indicate that the stirring speed has a negligible influence on the kinetics of crystallization of form II, but has a strong influence on the kinetics of transformation. Indeed, no substantial differences are noticed in the solute depletion kinetics at the beginning of the experiment, but a significantly longer plateau is observed when decreasing the stirring speed from $400 \mathrm{rpm}$ to $200 \mathrm{rpm}$. In addition, a higher concentration of form II is reached at lower stirring speeds.
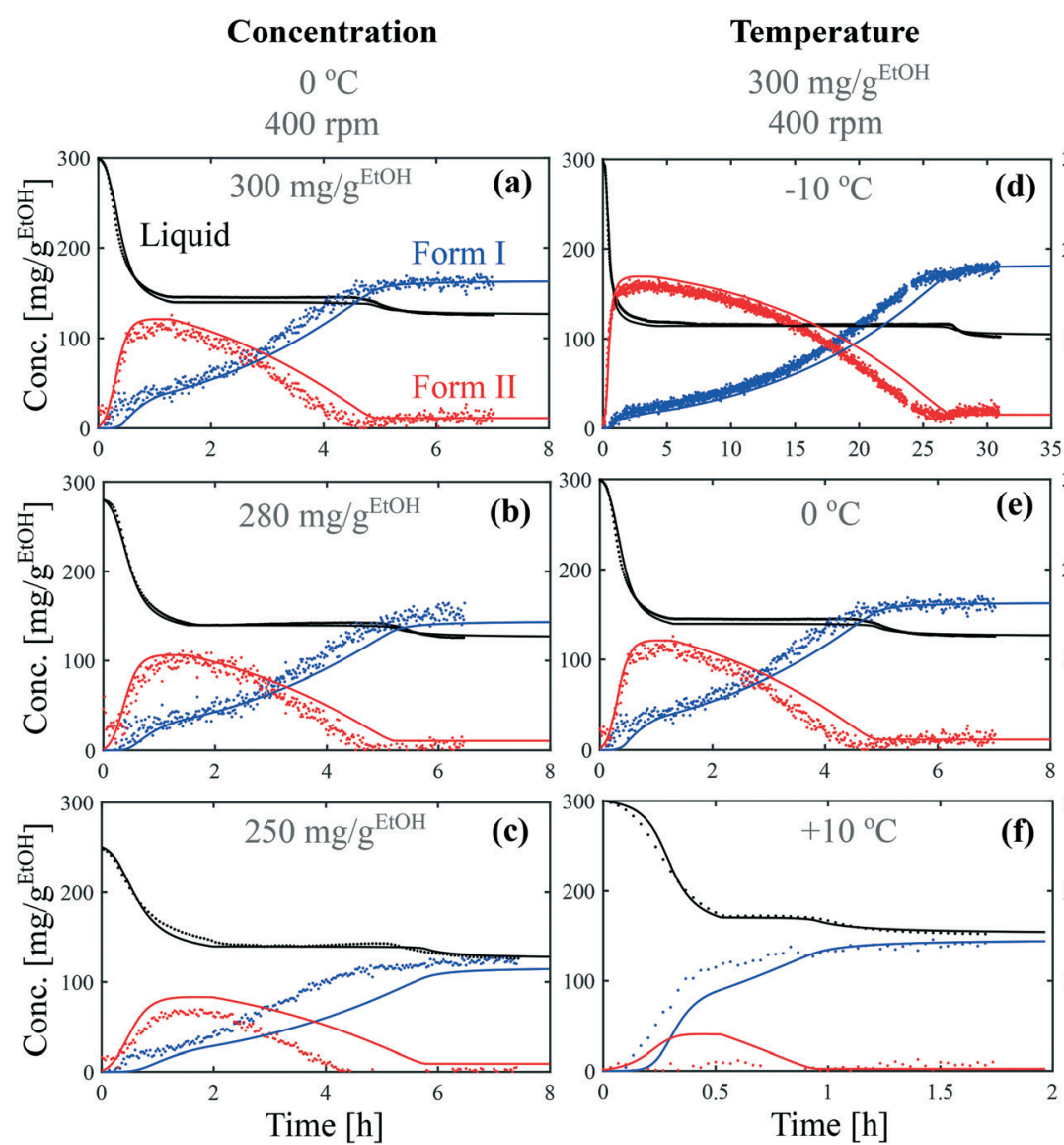

Agitation

$0{ }^{\circ} \mathrm{C}$

$300 \mathrm{mg} / \mathrm{g}^{\mathrm{EtOH}}$
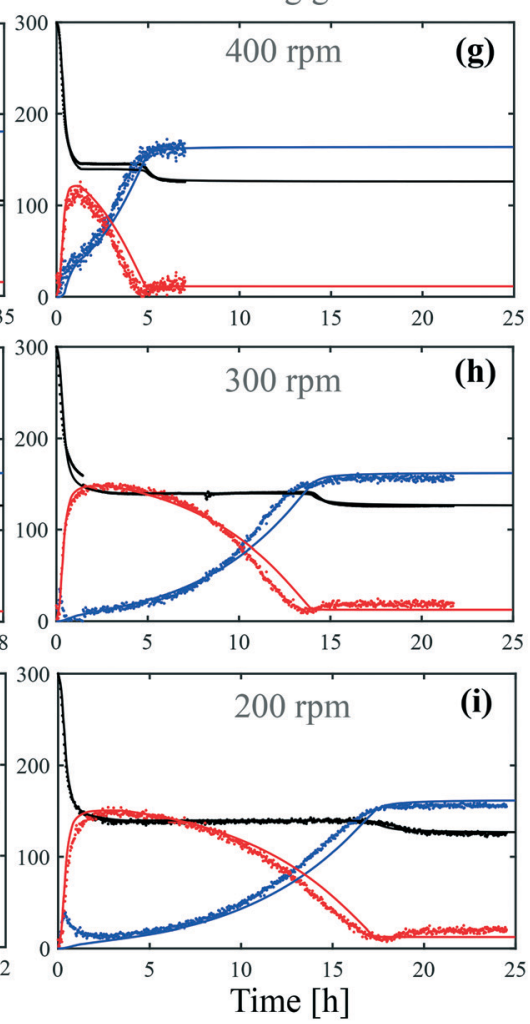

Fig. 5 Crystallization kinetic experiments under various conditions of (a-c) concentration, (d and e) temperature and, ( $\mathrm{g}-\mathrm{i}$ ) agitation speed. Experimental data are represented by dots and model simulations by lines. The concentrations of dissolved paracetamol, crystals of form I, and crystals of form II are show in black, blue, and red, respectively. The model parameters are summarized in Fig. 6 and 7. 


\subsection{Model parameters estimation}

In this section, we describe the experimental data of Fig. 5 with the kinetic model presented in section 1.6, which accounts for the primary (cross) nucleation, secondary nucleation, and growth of form I, as well as the secondary nucleation, growth and dissolution of form II. The implementation of the model requires first to estimate model parameters.

For the sake of simplicity, it was assumed that both polymorphs have the same density, which was set here to $\rho^{\mathrm{I}}=\rho^{\mathrm{II}}$ $=1332 \mathrm{~kg} \mathrm{~m}^{-3}$ in agreement with previous publications. ${ }^{34,42,43}$ The shape factor of form I has been estimated to $k_{\mathrm{v}}^{\mathrm{I}}=0.866$ in the literature. ${ }^{44}$ On the other hand, the shape factor of form II has been estimated in this work to $k_{\mathrm{v}}^{\mathrm{II}} \approx 2$. To do so, a simplified morphology based on the one reported in the literature $^{45}$ has been considered and pictures were analyzed to determine the length, width and thickness of a number of crystals of form II. The width was chosen as the characteristic length $L$, and the shape factor was estimated from the measurement of the crystal volume as $V=k_{\mathrm{v}} L^{3}$. More details about the calculation of the crystal volume as a function of the crystal width for the selected morphology are given in the ESI.†

The seed size distribution was described by a gamma distribution of average size and standard deviation equal to $120 \mu \mathrm{m}$ and $70 \mu \mathrm{m}$, respectively. The comparison between the experimental seed distribution estimated from online images and the fitted distribution is given in the ESI. $\dagger$

The parameters $n_{\mathrm{s}}$ and $n_{\mathrm{g}}$ were set to 3 and 2, respectively, so as to obtain a concentration dependence of the secondary nucleation rate and growth rate of form $I$ in reasonable agreement with previous works. ${ }^{34,43,44,46}$ In the absence of data on the cross-nucleation of form I over form II, several values of $n_{\mathrm{c}}$ ranging from 2 to 4 were tested. It was found that $n_{\mathrm{c}}=2$ describes best the experimental data.

The kinetic parameters $k_{\mathrm{c}}^{\mathrm{I}}, k_{\mathrm{s}}^{\mathrm{I}}, k_{\mathrm{g}}^{\mathrm{I}}, k_{\mathrm{s}}^{\mathrm{II}}, k_{\mathrm{g}}^{\mathrm{II}}$, and $k_{\mathrm{d}}^{\mathrm{II}}$ were determined by fitting model simulations to the experimental results obtained at the stirring speed of $400 \mathrm{rpm}$. For each temperature, at least two initial concentrations were considered. This corresponds to the data shown in Fig. 5(a-f) and in Fig. S11 of the ESI $\dagger$ (results at $250 \mathrm{mg} \mathrm{g}_{\mathrm{EtOH}}{ }^{-1}$ at $-10{ }^{\circ} \mathrm{C}$ and +10 $\left.{ }^{\circ} \mathrm{C}\right)$. The optimization procedure relied on the minimization of the sum of squared residuals with a genetic algorithm. The squared residuals of the solute concentration were multiplied by a factor 100 with respect to the squared residuals of the concentration of form II in order to obtain similar errors for both quantities. The model simulations are represented with solid lines in Fig. 5(a-f) and in Fig. S11 of the ESI, $\dagger$ showing a good agreement with the experimental data.

The parameters obtained for the reference case of $T=0{ }^{\circ} \mathrm{C}$ and $N=400 \mathrm{rpm}$ are presented in Table 2. For each kinetic parameter, the reported confidence interval corresponds to the range over which the considered parameter can be varied while leading to a change in the error function by less than $10 \%$ (keeping all the other parameters constant). It is observed that the growth rate constants are the parameters hav-
Table 2 Estimation of model parameters at $0^{\circ} \mathrm{C}$ and $400 \mathrm{rpm}$

\begin{tabular}{llll}
\hline & & Form I & Form II \\
\hline$n_{\mathrm{c}}$ & {$[-]$} & 2 & n.a. \\
$k_{\mathrm{c}}$ & {$\left[\# \mathrm{~m}^{-3} \mathrm{~min}\right]$} & $(1.37 \pm 0.13) \times 10^{7}$ & n.a. \\
$n_{\mathrm{s}}$ & {$[-]$} & 3 & 3 \\
$k_{\mathrm{s}}$ & {$\left[\# \mathrm{~m}^{-2} \mathrm{~min}\right]$} & $(7.17 \pm 0.23) \times 10^{9}$ & $(1.50 \pm 0.17) \times 10^{7}$ \\
$n_{\mathrm{g}}$ & {$[-]$} & 2 & 2 \\
$k_{\mathrm{g}}$ & {$\left[\mathrm{m} \mathrm{min}^{-1}\right]$} & $(1.04 \pm 0.01) \times 10^{-5}$ & $(5.22 \pm 0.07) \times 10^{-5}$ \\
$k_{\mathrm{d}}$ & {$\left[\mathrm{m} \mathrm{min}^{-1}\right]$} & n.a. & $(5.42 \pm 3.04) \times 10^{-4}$ \\
& & &
\end{tabular}

ing the smallest confidence intervals, then come the nucleation rate constants and finally the dissolution rate constant. The latter is difficult to estimate with accuracy because the polymorphic transformation of the investigated system is not limited by dissolution. In other words, any value of $k_{\mathrm{d}}^{\mathrm{II}}$ larger than a certain threshold ensures a satisfactory description of the experimental data. The crystallization of form II paracetamol from ethanolic solutions has already been reported, ${ }^{47,48}$ but to the best of our knowledge, no kinetic parameters related to the nucleation, growth and dissolution of form II are available to date in the literature. Nevertheless, the crystallization kinetics of form I has been studied by several authors. ${ }^{34,43,44,46}$ A comparison between the secondary nucleation and growth rates obtained in this work and those obtained in the aforementioned citations is provided in the ESI. $\uparrow$ The secondary nucleation rates obtained in the various studies span considerably different orders of magnitude, and the results obtained in this work fall in between the lowest and highest reported estimates. On the other hand, the data on the growth rates are quite consistent between the different works, this one included.

The temperature dependence of $k_{\mathrm{c}}^{\mathrm{I}}, k_{\mathrm{s}}^{\mathrm{I}}, k_{\mathrm{g}}^{\mathrm{I}}, k_{\mathrm{s}}^{\mathrm{II}}, k_{\mathrm{g}}^{\mathrm{II}}$, and $k_{\mathrm{d}}^{\mathrm{II}}$ can be assessed in Fig. 6. Those data were regressed with an Arrhenius model of the type $k=k_{0} \exp \left(-E_{\mathrm{a}} / R T\right)$, where $k_{0}$ is a prefactor, $E_{\mathrm{a}}$ the activation energy, and $R$ the ideal gas constant. The values of $k_{0}$ and $E_{\mathrm{a}}$ for the nucleation, growth and dissolution rate constants are summarized in Table 3. As mentioned previously, the data on the nucleation of form I are quite scattered in the literature. However, three separate studies report similar values of the activation energy of the growth of form I, namely around $41 \mathrm{~kJ} \mathrm{~mol}^{-1}$. $^{34,44,46}$ To compare the present results with those of these previous studies, it is first necessary to unify the definition of the reaction rates. Here, the reaction rates are written as:

$$
\mathscr{R}=k_{0} \exp \left(\frac{-E_{\mathrm{a}}}{R T}\right) \ln \left[\left(\frac{c^{\text {liq }}}{c^{*}}\right)\right]^{n}
$$

A different expression of the supersaturation was selected in previous works, so that the growth rates were written as: ${ }^{34,44,46}$

$$
\mathscr{R}=k_{0}^{\prime} \exp \left(\frac{-E_{\mathrm{a}}^{\prime}}{R T}\right)\left(c^{\text {liq }}-c^{*}\right)^{n}
$$



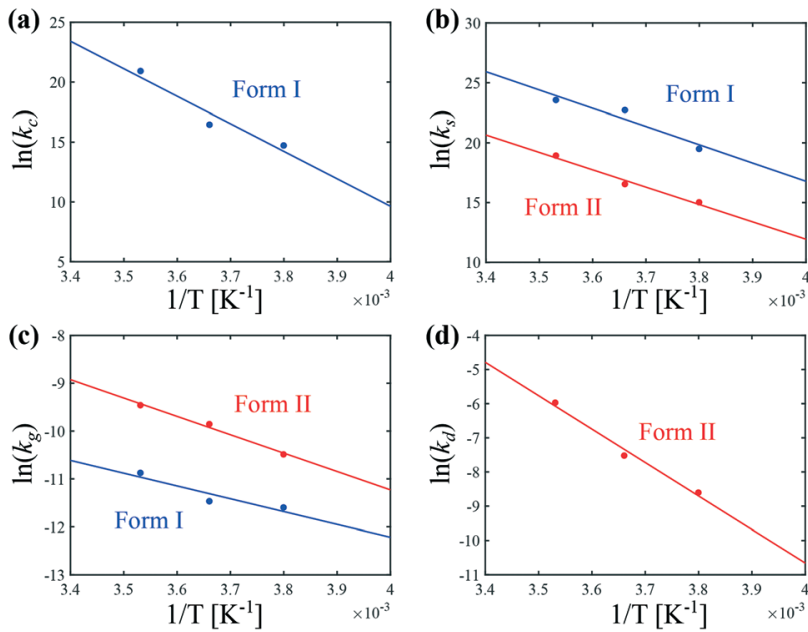

Fig. 6 Arrhenius dependence of the kinetic rate constants of (a) cross-nucleation, (b) secondary nucleation, (c) growth, and (d) dissolution at $400 \mathrm{rpm}$.

Table 3 Arrhenius dependence of kinetic parameters at $400 \mathrm{rpm}$

\begin{tabular}{lllll}
\hline & & & Form I & Form II \\
\hline$k_{\mathrm{c}}$ & $k_{\mathrm{o}}$ & {$\left[\# \mathrm{~m}^{-3} \mathrm{~min}\right]$} & $1.16 \times 10^{44}$ & n.a. \\
& $E_{\mathrm{a}}$ & {$\left[\mathrm{kJ} \mathrm{mol}^{-1}\right]$} & 191 & n.a. \\
$k_{\mathrm{s}}$ & $k_{\mathrm{o}}$ & {$\left[\# \mathrm{H}^{-2} \mathrm{~min}\right]$} & $5.62 \times 10^{33}$ & $2.53 \times 10^{30}$ \\
& $E_{\mathrm{a}}$ & {$\left[\mathrm{kJ} \mathrm{mol}^{-1}\right]$} & 127 & 121 \\
$k_{\mathrm{g}}$ & $k_{\mathrm{o}}$ & {$\left[\mathrm{m} \mathrm{min}^{-1}\right]$} & 0.21 & 62.7 \\
& $E_{\mathrm{a}}$ & {$\left[\mathrm{kJ} \mathrm{mol}^{-1}\right]$} & 22.2 & 31.9 \\
& $k_{\mathrm{d}}$ & {$\left[\mathrm{m} \mathrm{min}^{-1}\right]$} & n.a. & $2.34 \times 10^{12}$ \\
& $E_{\mathrm{a}}$ & {$\left[\mathrm{kJ} \mathrm{mol}^{-1}\right]$} & n.a. & 81.4
\end{tabular}

By approximating $\ln \left(c^{\mathrm{liq}} / c^{*}\right)$ to $\left(c^{\mathrm{liq}}-c^{*}\right) / c^{*}$, one obtains:

$$
E_{\mathrm{a}}^{\prime} \approx E_{\mathrm{a}}+R n \beta T^{2}
$$

where $\beta$ is a coefficient describing the temperature dependence of the solubility such that $\mathrm{c}^{*}=\alpha \exp (\beta T)$. By adapting eqn (18) (in mg $\mathrm{g}_{\mathrm{EtOH}}{ }^{-1}$ ) to the proper units $\left(\mathrm{kg} \mathrm{m}^{-3}\right.$ ), we obtain $\beta=0.016$. With $n=2, E_{\mathrm{a}}=22.2 \mathrm{~kJ} \mathrm{~mol}^{-1}$ and $T=0{ }^{\circ} \mathrm{C}$, it results that the activation energy of the growth of form $\mathrm{I}$ is in the order of $E_{\mathrm{a}}^{\prime}=42 \mathrm{~kJ} \mathrm{~mol}^{-1}$, which is in very good agreement with the value reported in previous works.

The data shown in Fig. 6 allow shedding light on the experimental observation that the crystallization of form II is favored at low temperatures (see Fig. 5). It is in fact seen in Fig. 6 that the primary (cross) nucleation of form I is strongly reduced at low temperatures. On the other hand, it seems that temperature affects the secondary nucleation and growth rates of forms I and II in a relatively similar way. Therefore, the inhibition of the crystallization of form I with respect to form II under cold conditions cannot be explained by a change in the ratios $k_{\mathrm{s}}^{\mathrm{I}} / k_{\mathrm{s}}^{\mathrm{II}}$ or $k_{\mathrm{g}}^{\mathrm{I}} / k_{\mathrm{g}}^{\mathrm{II}}$. Overall, the improvement in the content of form II at low temperatures is attributed to a decrease in the primary nucleation rate of form I. Note that for the sake of simplicity, the primary nucleation rate was considered here to be proportional to the surface of crystals of form II only, and was thus referred to as cross-nucleation. Nevertheless, the estimated kinetic constants rather correspond to lumped values considering all primary nucleation phenomena taken together, including cross-nucleation, but also homogeneous nucleation and heterogeneous nucleation on the surfaces present in the crystallizer (e.g., walls of the vessel, impeller, probes).

Let us now focus on the impact of the stirring speed. It was observed in Fig. 5(g-i) that a decrease in the stirring speed has a negligible impact on the decrease in the solute concentration before it reaches the solubility of form II, but significantly increases the length of the plateau at the solubility of form II. This suggests that the stirring speed has a weak impact on the crystallization of form II, but significantly delays the formation of form I. Accordingly, the parameters $k_{\mathrm{s}}^{\mathrm{II}}$ and $k_{g}^{\mathrm{II}}$ were kept unchanged at all stirring speeds. Under such vigorous stirring, it is unlikely that growth and dissolution are diffusion limited, so that $k_{\mathrm{g}}^{\mathrm{I}}$ and $k_{\mathrm{d}}^{\mathrm{II}}$ can also reasonably be considered independent of the stirring speed. Overall, a change in the stirring speed is expected to impact $k_{\mathrm{c}}^{\mathrm{I}}$ and/or $k_{\mathrm{s}}^{\mathrm{I}}$. For the sake of simplicity, it was assumed that only $k_{\mathrm{s}}^{\mathrm{I}}$ is impacted by the stirring speed. The fitted values of $k_{\mathrm{s}}^{\mathrm{I}}$ are plotted as a function of $N$ in Fig. 7 (symbols), while the quality of the fitting can be appreciated in Fig. $5(\mathrm{~g}-\mathrm{i})$. It is seen that the experimental data acquired between $200 \mathrm{rpm}$ and $400 \mathrm{rpm}$ can be well explained by a change in $k_{\mathrm{s}}^{\mathrm{I}}$ only. The trend of $k_{\mathrm{s}}^{\mathrm{I}}$ with $N$ can be described by the following empirical expression: $k_{\mathrm{s}}^{\mathrm{I}}=106 N^{3}$, which is represented by a solid line in Fig. 7. Therefore, the effect of temperature and agitation speed on $k_{\mathrm{s}}^{\mathrm{I}}$ can be accounted for simultaneously using the following lumped expression:

$$
k_{\mathrm{s}}^{\mathrm{I}}=k_{0}^{\prime}\left(\frac{N}{N_{\text {ref }}}\right)^{3} \exp \left(-\frac{E_{\mathrm{a}}}{R T}\right)
$$

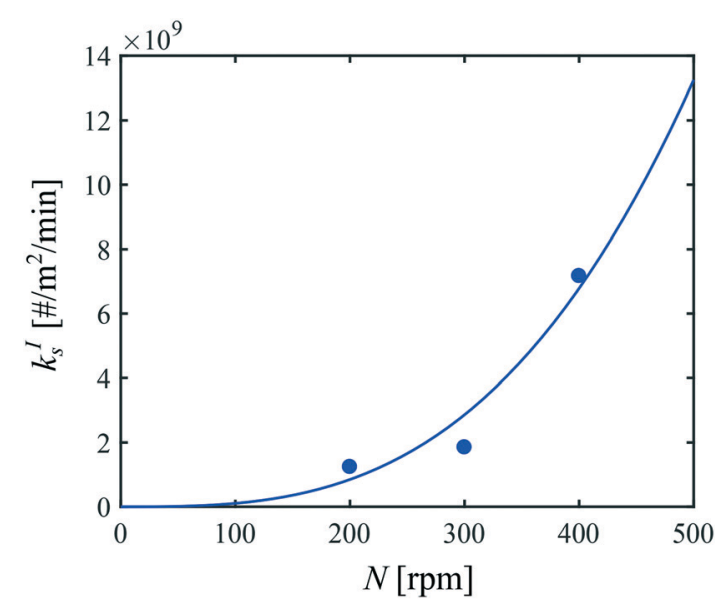

Fig. 7 Impact of the agitation speed on the secondary nucleation rate constant of form I at $0{ }^{\circ} \mathrm{C}$. Symbols correspond to fitted values of $k_{\mathrm{s}}^{\prime}$, while the line represents the following empirical expression $k_{\mathrm{s}}^{1}=$ $106 N^{3}$. 
with $k_{0}^{\prime}=8.6 \times 10^{25} \# \mathrm{~m}^{-2} \min$ and $E_{\mathrm{a}}=127 \mathrm{~kJ} \mathrm{~mol}^{-1}$. The reference stirring speed $N_{\text {ref }}$ is set to $1 \mathrm{rpm}$ so as to have $k_{\mathrm{s}}^{\mathrm{I}}$ and $k_{0}^{\prime}$ in the same units.

\subsection{Process performance parameters}

The kinetic model developed in the previous section can now be used to select the operating conditions allowing the production of form II within some target specifications in terms of polymorphic purity, yield, and productivity. These three process performance parameters are defined as follows:

Polymorphic purity: mass of form II over total mass of crystals

$$
\chi=\frac{c^{\mathrm{II}}}{c^{\mathrm{I}}+c^{\mathrm{II}}}
$$

Yield: produced mass of form II over total mass of paracetamol

$$
\vartheta=\chi \times \frac{c_{0}^{\text {liq }}-c^{\text {liq }}}{c_{0}^{\text {liq }}}
$$

Productivity: mass of crystals per unit volume and unit time

$$
\varphi=\frac{c_{0}^{\mathrm{liq}}-c^{\mathrm{liq}}}{\Delta t}
$$

where $\Delta t$ is the time elapsed after seeding the crystallizer.
The impact of concentration, temperature, and agitation on the polymorphic purity, yield, and productivity is analyzed in Fig. 8. Let us start with Fig. 8(a), which shows the yield as a function of the polymorphic purity for various initial concentrations. For each of these curves, at time 0 , the polymorphic purity is equal to $100 \%$ and the yield to $0 \%$ because it corresponds to the presence of the seeds only. When time passes, crystals are formed, the yield increases, but the polymorphic purity decreases due to the crystallization of form I. When the yield is maximum, the solubility of form II is reached. Later on, the yield decreases due to solventmediated transformation. Therefore, crystals should be harvested before the yield starts decreasing, which means that only the right part of the curves shown in Fig. 8 should be considered for process design. It is observed in Fig. 8(a) that an increase in the initial concentration leads to an increase in the yield. To understand this observation, let us introduce the theoretical yield defined as follows:

$$
\vartheta *=\frac{c_{0}^{\mathrm{liq}}-c_{\mathrm{II}}^{*}}{c_{0}^{\mathrm{liq}}}
$$

The theoretical yield corresponds to the "best possible yield", i.e., the yield that would be obtained if form II could be crystallized at $100 \%$ purity when the solute concentration reaches the solubility of form II. When the initial concentration is increased from $250 \mathrm{mg} \mathrm{g}_{\mathrm{EtOH}}{ }^{-1}$ to $350 \mathrm{mg} \mathrm{g}_{\mathrm{EtOH}}{ }^{-1}$, the
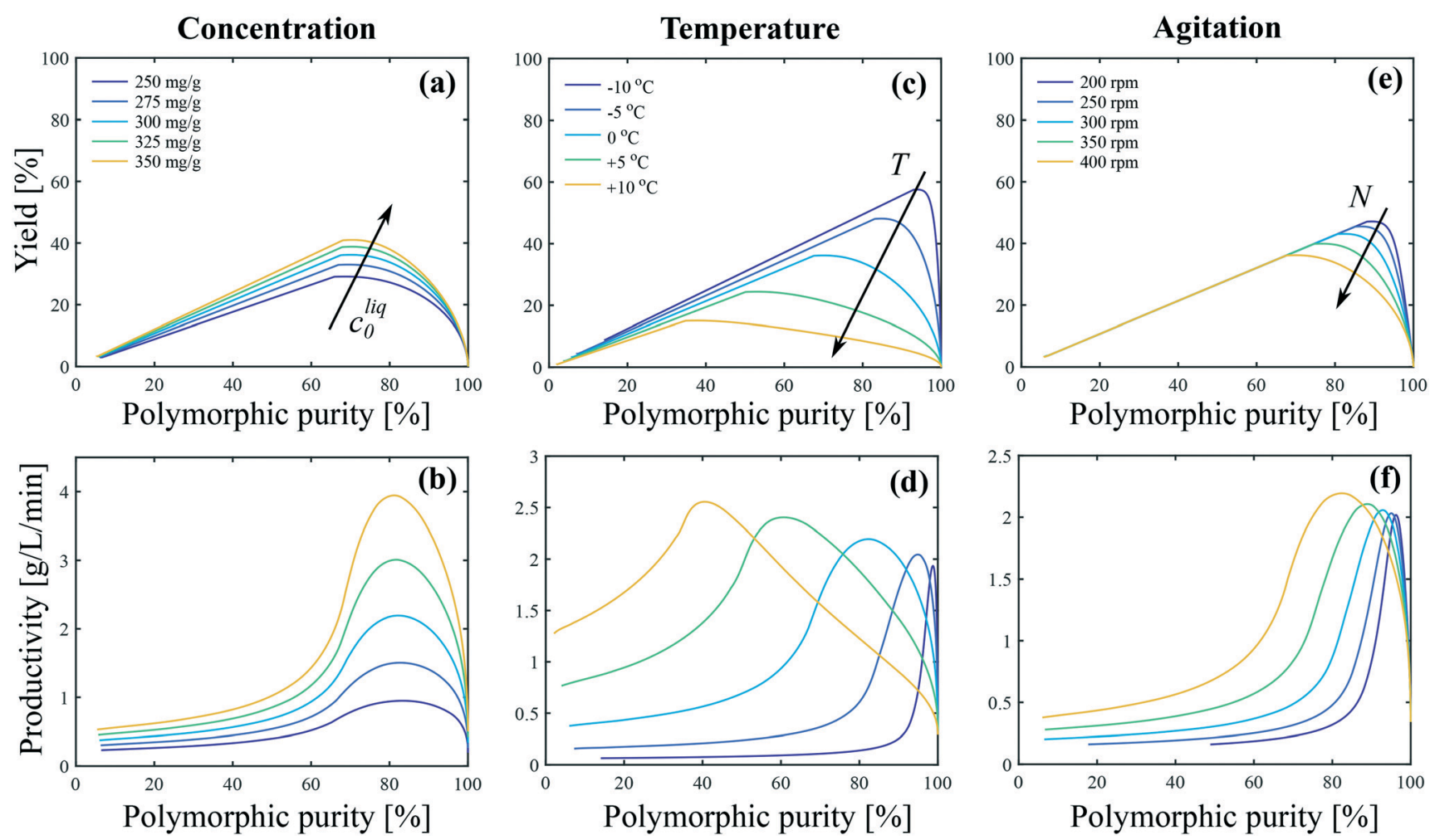

Fig. 8 Impact of (a and b) concentration, (c and d) temperature, and (e and f) agitation on the polymorphic purity, yield and productivity. The process performance parameters are defined with respect to form II as shown in eqn (25)-(27). In panels (a and b) $T=0{ }^{\circ} \mathrm{C}$ and $N=400 \mathrm{rpm}$. In panels (c and d) $C_{0}^{\text {liq }}=300 \mathrm{mg} \mathrm{g}_{\mathrm{EtOH}}{ }^{-1}$ and $N=400 \mathrm{rpm}$. In panels (e and f) $T=0{ }^{\circ} \mathrm{C}$ and $C_{0}^{\text {liq }}=300 \mathrm{mg} \mathrm{g}_{\mathrm{EtOH}}{ }^{-1}$. 
theoretical yield increases from $44 \%$ to $60 \%$. On the other hand, the maximum achievable yield increases from $29 \%$ to $41 \%$ (see Fig. 8(a)), so that $\vartheta / \vartheta^{*}$ increases from approximately $66 \%$ to $68 \%$. This is better shown in Fig. S13(a) of the ESI, $\dagger$ where the ratio $\vartheta / \vartheta^{*}$ is plotted as a function of the polymorphic purity. It is seen that the curves obtained at various initial concentrations all overlap on one single curve. This indicates that the improvement in the yield-purity trade-off observed in Fig. 8(a) at higher concentration is mainly due to an increase in the theoretical yield. Considering now Fig. 8(b), the productivity starts at 0 (when the polymorphic purity is $100 \%$ ) and increases due to the formation of crystals. When the solubility of form II is reached, solutionmediated transformation starts. No more crystals are produced and the productivity becomes inversely proportional to time. It is observed in Fig. 8(b) that an increase in $c_{0}^{\text {liq }}$ leads to an increase in productivity. This is because, for a given reactor volume, productivity is directly proportional to the concentration of crystals produced.

Let us now focus on the impact of temperature. It is observed in Fig. 8(c) that temperature has a strong impact on the yield-purity trade-off. Indeed, it is possible to obtain simultaneously a high yield and a high polymorphic purity only at low temperatures. For instance, if a polymorphic purity of $95 \%$ is targeted, the yield at $+10{ }^{\circ} \mathrm{C}$ is only in the order of $5 \%$, whereas it is around $60 \%$ at $-10{ }^{\circ} \mathrm{C}$. When the temperature is decreased from $+10{ }^{\circ} \mathrm{C}$ to $-10{ }^{\circ} \mathrm{C}$, the theoretical yield increases from $43 \%$ to $62 \%$. On the other hand, the maximum achievable yield increases from $15 \%$ to $58 \%$ (Fig. 8 (b)). Consequently, the ratio $\vartheta / \vartheta^{*}$ increases from $35 \%$ to $93 \%$ when decreasing the temperature from $+10{ }^{\circ} \mathrm{C}$ to $-10{ }^{\circ} \mathrm{C}$. This is further illustrated in Fig. S13(b) of the ESI, $†$ where the ratio $\vartheta / \vartheta^{*}$ is plotted as a function of the polymorphic purity for various temperatures. The improvement in the yield-purity trade-off observed in Fig. 8(c) at low temperatures is thus mainly due to the inhibition of primary nucleation of form I (and also to an increase in the theoretical yield). However, such improvement of the yield comes at the expense of a lower productivity because lower temperatures are associated with slower kinetics. This is illustrated in Fig. 8(d), where it is seen that the productivity maximum decreases when the temperature is decreased. In addition, very cold temperatures may be difficult to achieve in large scale crystallizers and are energy intensive.

Finally, the impact of agitation can be appreciated in Fig. 8(e and f). It is observed that, for a given target purity, a higher yield is obtained at low stirring speed. This is due to the inhibition of the crystallization of form I with respect to form II at lower stirring speeds. Nevertheless, a sufficient stirring speed should be selected in order to keep the crystals suspended and ensure a good mass/heat transfer.

It is evident from Fig. 8 that all process performance parameters (i.e., polymorphic purity, yield, and productivity) cannot be maximized simultaneously. Therefore, depending on the process specifications, different operating conditions should be selected. This is illustrated in Fig. 9, where the pro-

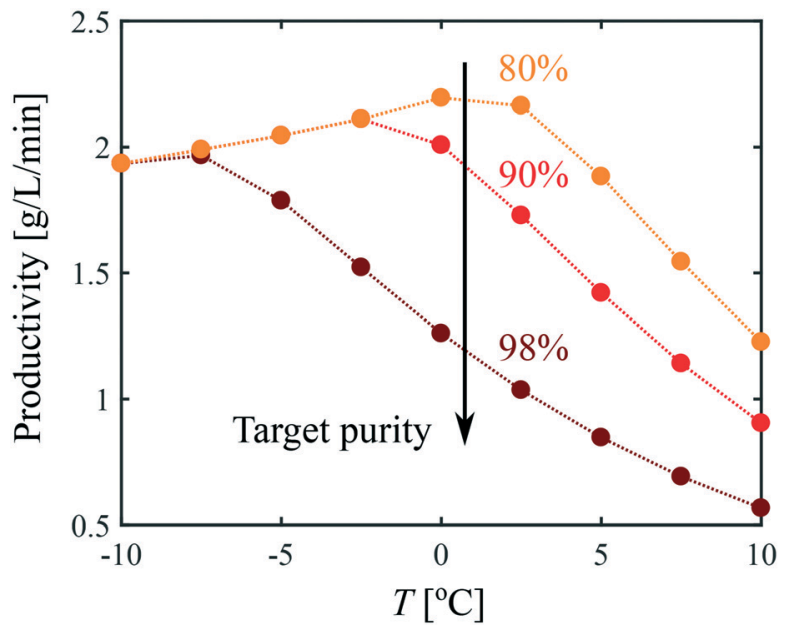

Fig. 9 productivity as a function of temperature for different target purity values.

ductivity is plotted as a function of the temperature for different values of the target polymorphic purity. For instance, it is seen that if a rather low polymorphic purity is acceptable (e.g., 80\%), the productivity goes through a maximum as a function of temperature, and the most productive process is obtained at around $0{ }^{\circ} \mathrm{C}$. On the other hand, if a very high polymorphic purity is requested (e.g., 98\%) and the process is still operated at $0{ }^{\circ} \mathrm{C}$, the crystallization should be stopped prematurely to satisfy the purity constraint, which would lead to a low productivity. Therefore, if a high polymorphic purity is targeted, it is necessary to run the process at very low temperatures, in the order of $-10^{\circ} \mathrm{C}$. These results illustrate how process modeling can help selecting the most appropriate operating conditions for a given system.

\section{Conclusions}

In this work, we investigated polymorph dynamics in seeded batch crystallizers considering the cooling crystallization of paracetamol form II as a case study. The solute concentration and the polymorphic content were monitored with online ATR-FTIR and online Raman spectroscopy, respectively. A proper calibration of the Raman signal could be obtained by applying principal component regression on only one kinetic experiment, where form II crystallizes and then transforms into form I. A suitable pre-processing treatment of the spectra was found necessary to obtain a satisfactory calibration.

Experimental characterization was complemented by population balance modeling considering the primary (cross) nucleation, secondary nucleation, and growth of form I, as well as the secondary nucleation, growth, and dissolution of form II. It was found that low temperatures and low agitation speeds promote the crystallization of form II with respect to form I due to a decrease in the nucleation rate of form I. In particular, it was observed that at temperatures above approximately $0{ }^{\circ} \mathrm{C}$, seeding the crystallizer with crystals of form II is not sufficient to induce the crystallization of form II. 
The impact of operating conditions (concentration, temperature, stirring speed) on process performance parameters (polymorphic purity, yield, productivity) was investigated. It was shown that the temperature leading to the most productive process strongly depends on the target polymorphic purity. This work illustrates how the combination of experimental characterization with mechanistic modeling allows gaining a deep understanding of the crystallization process and selecting the most suited operating conditions to satisfy certain process requirements.

\section{Conflicts of interest}

There are no conflicts to declare.

\section{Acknowledgements}

Financial support from the Swiss National Science Foundation is gratefully acknowledged (grant P2EZP2_168909). We thank Richard Becker for interesting discussions and valuable technical support.

\section{References}

1 H. G. Brittain, Polymorphism in pharmaceutical solids, CRC Press, 2016.

2 A. Y. Lee, D. Erdemir and A. S. Myerson, Annu. Rev. Chem. Biomol. Eng., 2011, 2, 259-280.

3 E. H. Lee, Asian J. Pharm. Sci., 2014, 9, 163-175.

4 M. Kitamura, CrystEngComm, 2009, 11, 949-964.

5 A. Llinàs and J. M. Goodman, Drug Discovery Today, 2008, 13, 198-210.

6 D. Mangin, F. Puel and S. Veesler, Org. Process Res. Dev., 2009, 13, 1241-1253.

7 Z. Yu, J. Chew, P. Chow and R. Tan, Chem. Eng. Res. Des., 2007, 85, 893-905.

8 J. F. B. Black, P. T. Cardew, A. J. Cruz-Cabeza, R. J. Davey, S. E. Gilks and R. A. Sullivan, CrystEngComm, 2018, 20, 768-776.

9 J. Cornel, P. Kidambi and M. Mazzotti, Ind. Eng. Chem. Res., 2010, 49, 5854-5862.

10 J. Cornel, C. Lindenberg and M. Mazzotti, Ind. Eng. Chem. Res., 2008, 47, 4870-4882.

11 J. Schöll, D. Bonalumi, L. Vicum, M. Mazzotti and M. Müller, Cryst. Growth Des., 2006, 6, 881-891.

12 R. Davey, N. Blagden, S. Righini, H. Alison and E. Ferrari, J. Phys. Chem. B, 2002, 106, 1954-1959.

13 K. Sato, J. Phys. D: Appl. Phys., 1993, 26, B77.

14 E. S. Ferrari and R. J. Davey, Cryst. Growth Des., 2004, 4, 1061-1068.

15 C. Gu, V. Young Jr and D. J. Grant, J. Pharm. Sci., 2001, 90, 1878-1890.

16 N. Guo, B. Hou, N. Wang, Y. Xiao, J. Huang, Y. Guo, S. Zong and H. Hao, J. Pharm. Sci., 2018, 107, 344-352.

17 G. Févotte, A. Caillet and N. Sheibat-Othman, AIChE J., 2007, 53, 2578-2589.
18 W. Beckmann, Org. Process Res. Dev., 2000, 4, 372-383.

19 M. Müller, U. Meier, D. Wieckhusen, R. Beck, S. PfefferHennig and R. Schneeberger, Cryst. Growth Des., 2006, 6, 946-954.

20 J. Bauer, S. Spanton, R. Henry, J. Quick, W. Dziki, W. Porter and J. Morris, Pharm. Res., 2001, 18, 859-866.

21 P. Di Martino, A. Guyot-Hermann, P. Conflant, M. Drache and J. Guyot, Int. J. Pharm., 1996, 128, 1-8.

22 E. Joiris, P. Di Martino, C. Berneron, A. M. Guyot-Hermann and J. C. Guyot, Pharm. Res., 1998, 15, 1122-1130.

23 P. Di Martino, P. Conflant, M. Drache, J.-P. Huvenne and A.-M. Guyot-Hermann, J. Therm. Anal., 1997, 48, $447-458$.

24 S. J. Smith, M. M. Bishop, J. M. Montgomery, T. P. Hamilton and Y. K. Vohra, J. Phys. Chem. A, 2014, 118, 6068-6077.

25 L. R. Agnew, D. L. Cruickshank, T. McGlone and C. C. Wilson, Chem. Commun., 2016, 52, 7368-7371.

26 S. Chen, H. Xi and L. Yu, J. Am. Chem. Soc., 2005, 127, 17439-17444.

27 C. Cashell, D. Corcoran and B. Hodnett, Chem. Commun., 2003, 374-375.

28 J. Tao and L. Yu, J. Phys. Chem. B, 2006, 110, 7098-7101.

29 J. Tao, K. J. Jones and L. Yu, Cryst. Growth Des., 2007, 7, 2410-2414.

30 D. Cavallo, F. Galli, L. Yu and G. C. Alfonso, Cryst. Growth Des., 2017, 17, 2639-2645.

31 S. Qamar, G. Warnecke, M. P. Elsner and A. SeidelMorgenstern, Chem. Eng. Sci., 2008, 63, 2233-2240.

32 L. Nicoud, F. Licordari and A. S. Myerson, Cryst. Growth Des., 2018, DOI: 10.1021/acs.cgd.8b01200.

33 L. L. Simon, H. Pataki, G. Marosi, F. Meemken, K. Hungerbühler, A. Baiker, S. Tummala, B. Glennon, M. Kuentz and G. Steele, Org. Process Res. Dev., 2015, 19, 3-62.

34 N. A. Mitchell, C. T. Ó'Ciardhá and P. J. Frawley, J. Cryst. Growth, 2011, 328, 39-49.

35 A. Maher, Ã. C. Rasmuson, D. M. Croker and B. K. Hodnett, J. Chem. Eng. Data, 2012, 57, 3525-3531.

36 N. Al-Zoubi, J. Koundourellis and S. Malamataris, J. Pharm. Biomed. Anal., 2002, 29, 459-467.

37 C. Starbuck, A. Spartalis, L. Wai, J. Wang, P. Fernandez, C. M. Lindemann, G. X. Zhou and Z. Ge, Cryst. Growth Des., 2002, 2, 515-522.

38 G. Févotte, Chem. Eng. Res. Des., 2007, 85, 906-920.

39 E. Simone, A. Saleemi and Z. Nagy, Chem. Eng. Res. Des., 2014, 92, 594-611.

40 Å. Rinnan, F. van den Berg and S. B. Engelsen, TrAC, Trends Anal. Chem., 2009, 28, 1201-1222.

41 J. Huang, S. Romero-Torres and M. Moshgbar, Am. Pharm. Rev., 2010, 13, 116.

42 T. Vetter, C. L. Burcham and M. F. Doherty, Chem. Eng. Sci., 2014, 106, 167-180.

43 P. J. Frawley, N. A. Mitchell, C. T. Ó'Ciardha and K. W. Hutton, Chem. Eng. Sci., 2012, 75, 183-197.

44 J. Worlitschek and M. Mazzotti, Cryst. Growth Des., 2004, 4, 891-903. 
45 G. Nichols and C. S. Frampton, J. Pharm. Sci., 1998, 87, 684-693.

46 H. Li, Y. Kawajiri, M. A. Grover and R. W. Rousseau, Ind. Eng. Chem. Res., 2017, 56, 4060-4073.
47 N. Al-Zoubi, K. Kachrimanis and S. Malamataris, Eur. J. Pharm. Sci., 2002, 17, 13-21.

48 N. Al-Zoubi, I. Nikolakakis and S. Malamataris, J. Pharm. Pharmacol., 2002, 54, 325-333. 